\title{
Estudo da Associação entre Características de Desempenho e de Carcaça de Suínos por Meio de Correlação Canônica ${ }^{1}$
}

\section{Leandro Barbosa ${ }^{2}$, Paulo Sávio Lopes ${ }^{3}$, Adair José Regazzi ${ }^{4}$, Simone Eliza Facioni Guimarães ${ }^{5}$, Robledo de Almeida Torres ${ }^{6}$}

\begin{abstract}
RESUMO - Dados de 844 animais de uma população F2 de suínos foram utilizados para avaliar a associação entre características de desempenho e carcaça por meio de correlação canônica. Foram consideradas no estudo, além das características de desempenho, o tamanho da leitegada ao nascimento, o número de tetos, o peso aos 77 dias de idade, o consumo de ração e a conversão alimentar dos 77 aos 105 dias e as seguintes características de carcaça: idade ao abate; peso da meia-carcaça direita; comprimento de carcaça pelo Método Brasileiro de Classificação de Carcaça; maior espessura de toucinho na região da copa, na linha dorso-lombar; profundidade de lombo; e pesos do coração, do baço, do rim, da meia-carcaça direita resfriada, do pernil, da copa, da paleta, do carré, das costelas, da papada e do filezinho. Observou-se que os dois grupos de características não são independentes, pois apresentaram correlacão significativa, visto que a correlação do primeiro par canônico (r) foi de 0,7804. Os dois primeiros pares canônicos foram estatisticamente relevantes. No exame das variáveis que compõem o primeiro par canônico, para as características de desempenho, houve predomínio absoluto da variável peso aos 77 dias de idade; para as características de carcaça, a variável idade ao abate teve predomínio absoluto. Portanto, maior peso aos 77 dias de idade proporciona menor idade ao abate.
\end{abstract}

Palavras-chave: análise multivariada, carcaça, correlação, desempenho, pares canônicos, variáveis canônicas

\section{Association Between Performance and Carcass Traits of Swine Using Canonical Correlation}

\begin{abstract}
Records of 844 animals of a F2 swine population were used to evaluate the association among four performance traits (litter size at birth, teat number, weight at 77 days of age, feed intake and feed:gain ratio from 77 to 105 days of age) and 17 carcass traits (slaughter age, right side carcass weight, carcass length by the Brazilian Carcass Classification Method, backfat thickness at boston shoulder area and the midline, loin depth, heart, spleen, kidney, cold right side carcass, ham, boston shoulder, picnic shoulder, loin (bone-in), spareribs, jowl and sirloin weights) using canonical correlation analysis. The first canonical correlation (r) was 0.7804 , indicating performance and carcass traits were not independent. The first two canonical variable pairs were statistically significant. In the first canonical variable pair, weight at 77 days of age and slaughter age were the absolute predominant traits. These results suggest larger weight at 77 days indicates reduced age at slaughter.
\end{abstract}

Key Words: canonical variables, canonical variable pair, carcass, correlation, multivariate analysis, performance

\section{Introdução}

O melhoramento animal, ao longo de várias gerações, altera a composição gênica dos indivíduos, visando atender à demanda dos produtores e consumidores e, no melhoramento de suínos, o principal objetivo é a melhoria no desempenho, no rendimento de carcaça e na qualidade da carne.

Considerando-se que as características de importância econômica, utilizadas para avaliar os indivíduos ou genótipos, são de natureza quantitativa e, portanto, sujeitas a diferentes bases genéticas e graus de influên- cia de ambiente, torna-se difícil interpretar o conjunto de características a partir de análises univariadas (Sakaguti et al., 1996). Para estudos em que várias variáveis devam ser consideradas simultaneamente, as técnicas de análise multivariada são métodos estatísticos mais apropriados (Franci et.al., 2001). Para muitos dados biológicos, a correlação entre as variáveis é comum (Roso et al., 1995). Assim, as informações resultantes de análises univariadas isoladas podem ser redundantes quando em um complexo de variáveis. As técnicas de análise multivariada combinam, simultaneamente, as informações múltiplas, podendo-se

\footnotetext{
${ }^{1}$ Parte da Dissertação apresentada à UFV, pelo primeiro autor, para obtenção do grau de "Magister Scientiae" em Zootecnia. ${ }^{2}$ Estudante de Pós-Graduação da UFV, Av. P.H. Rolfs, s/n, Viçosa-MG, CEP: 36570-000 (leandro.b@vicosa.ufv.br). ${ }^{3}$ Professor do Departamento de Zootecnia, UFV, Av. P.H. Rolfs, s/n, Viçosa-MG - CEP: 36570-000 (plopes@ufv.br). ${ }^{4}$ Professor do Departamento de Informática, UFV, Av. P.H. Rolfs, s/n, Viçosa-MG - CEP: 36570-000 (adairreg@ufv.br) ${ }^{5}$ Professor do Departamento de Zootecnia, UFV, Av. P.H. Rolfs, s/n, Viçosa-MG - CEP: 36570-000 (sfacioni@ufv.br). ${ }^{6}$ Professor do Departamento de Zootecnia, UFV, Av. P.H. Rolfs, s/n, Viçosa-MG - CEP: 36570-000 (rtorres@ufv.br).
} 
chegar a conclusões impossíveis de serem obtidas com uso da estatística univariada (Liberato, 1995; Liberato et al., 1999).

A associação entre características de interesse econômico assume importância fundamental no melhoramento genético, principalmente no estudo das respostas correlacionadas, que são alterações provocadas em algumas características pela mudança em outras. Essas respostas correlacionadas podem ser vantajosas ou não, e de magnitudes variadas (Sakaguti, 1994).

Em suínos, foram encontradas correlações positivas entre ganho de peso diário e espessura de toucinho, por Costa et al. (2001), e negativas entre idade, em determinado peso, e espessura de toucinho, por Silva et al. (1992), Torres Filho (2001) e Torres Jr. et al. (1998). As correlações genéticas entre espessura de toucinho e características de carcaça são negativas (Almeida Neto et al., 1993; Roso et al., 1995; Silva et al., 1992), o que é favorável à seleção apenas para redução na espessura de toucinho. A associação desfavorável entre características geralmente causa atraso no progresso genético quando há seleção para cada característica separadamente, razão pela qual devem-se utilizar metodologias ou procedimentos multivariados (Costa et al., 2001).

Identificar e quantificar essa associação entre características de desempenho e de carcaça é importante para promover seleção mais eficiente, visando atender aos objetivos do melhoramento genético de suínos. Um método que possibilita o estudo dessa associação entre dois conjuntos de variáveis é a análise de correlação canônica (Nathier-Dufour et al., 1995).

A análise de correlação canônica é utilizada no intuito de identificar e quantificar a associação entre dois conjuntos de variáveis (Johnson \& Wichern, 1998). O coeficiente de correlação canônica, para cada par canônico, mede a intensidade da correlação, enquanto a composição das variáveis canônicas exprime a natureza da associação.

Objetivou-se neste trabalho identificar e quantificar a associação entre as características de desempenho e de carcaça em suínos.

\section{Material e Métodos}

Os dados utilizados neste estudos são referentes a uma geração F2 de suínos, desenvolvida no Departamento de Zootecnia da Universidade Federal de
Viçosa, em Viçosa - MG, no período de novembro de 1998 a julho de 2001.

Foram formadas duas famílias, resultantes do cruzamento de dois varrões da raça nativa brasileira Piau com 18 fêmeas de linhagem desenvolvida na UFV, pelo acasalamento de animais das raças Landrace, Large White e Pietran. A geração F1 foi acasalada inter si para produção da geração F2, que nasceu entre junho de 2000 e fevereiro de 2001. Assim, foram obtidos 844 animais F2, divididos em cinco lotes, segundo a data de nascimento: lote 1 (20/ 06 a 03/07/00), lote 2 (03/08 a 23/08/00), lote 3 (16/09 a $01 / 11 / 00)$, lote $4(30 / 11$ a $25 / 12 / 00)$ e lote $5(19 / 01$ a $12 / 02 / 01)$.

Ao nascimento, os animais foram identificados individualmente, utilizando-se o sistema de marcação australiano (mossagem nas orelhas), pesados e submetidos ao corte de dentes e à aplicação de antibiótico. Entre o 3o e 5을 dias, foi aplicada uma dose (3 mL) de ferro injetável, para se prevenir anemia. Os machos foram castrados por volta do $10^{\circ}$ dia de idade, quando foi disponibilizada ração pré-inicial aos leitões. Os animais foram desmamados aos 21 dias de idade, novamente pesados, receberam mais uma dose $(3 \mathrm{~mL})$ de ferro injetável e foram transferidos para a creche, onde permaneceram até aproximadamente 60 dias de idade. Após esse período, foram transferidos para o setor de cria/recria, onde ficaram até os 77 dias de idade, quando se iniciou o teste de conversão alimentar. O teste foi conduzido em galpão, dotado de baias individuais, onde os animais foram mantidos durante 28 dias (77 a 105 dias de idade).

Foram avaliados 844 animais para características de desempenho, dos quais 550 (fêmeas e machos castrados) foram abatidos aos 64,79 \pm 5,06 kg de peso vivo.

Os animais foram abatidos na granja em que foram criados. Após jejum de 18 horas, com livre acesso à água fresca, foram conduzidos à sala de abate e submetidos à insensibilização elétrica. A sangria foi realizada imediatamente após a insensibilização, pela punção do coração, por meio de inserção sob a axila esquerda do animal.

A seguir, os animais foram chamuscados e as cerdas foram manualmente raspadas com faca sob fluxo de água. As carcaças foram suspensas pelas patas traseiras, eventradas, evisceradas, lavadas, serradas longitudinalmente, inclusive a cabeça, e pesadas. A seguir, foram resfriadas, em freezers horizontais a $4^{\circ} \mathrm{C}$, por 24 horas. 
Preliminarmente, foi realizado o teste do número de condições citado por Montgomery \& Peck (1992), para diagnóstico do efeito da multicolinearidade ou dependência linear entre as variáveis, que pode levar à formação de matrizes singulares ou mal condicionadas. Após essa análise, por terem provocado forte multicolinearidade, foram identificadas e descartadas, entre as características de desempenho, a variável peso aos 105 dias de idade e, entre as características de carcaça, as seguintes variáveis: peso da meia-carcaça esquerda, peso da carcaça, peso ao abate, menor espessura de toucinho na região acima da última vértebra lombar, na linha dorsolombar, e espessura de toucinho a $6,5 \mathrm{~cm}$ da linha dorso lombar, equivalente a $\mathrm{P}_{2}$.

As características avaliadas foram previamente submetidas à análise de componentes principais para eliminação das variáveis redundantes, de modo que das características de desempenho e carcaça, 54,54 e $51,51 \%$, respectivamente, foram descartadas, conforme descrito por Barbosa (2003), uma vez que, na análise de correlações canônicas, cada conjunto de variáveis deve ser linearmente independente (Khattree \& Naik, 2000; Regazzi, 2002).Vainionpãã et al. (2000) e Dufour et al. (2001), para obterem variáveis linearmente independentes em seus conjuntos de dados, utilizaram a análise de componentes principais para, posteriormente, empregarem a análise de correlação canônica.

Neste estudo, foram avaliadas as seguintes características de desempenho: tamanho da leitegada ao nascimento (TLN), número de tetos (NT), peso aos 77 dias de idade (P77), consumo de ração (CR) e conversão alimentar dos 77 aos 105 dias (CA). Avaliaram-se as seguintes características de carcaça: idade ao abate (IDA); peso da meia-carcaça direita (PBDIR); comprimento de carcaça pelo Método Brasileiro de Classificação de Carcaça (CCMB); maior espessura de toucinho na região da copa, na linha dorso-lombar (SH); profundidade de lombo (PROFLOMB); e pesos do coração (COR), do baço (BACO), do rim (RIM), da meia-carcaça direita resfriada (PBDIRres), do pernil (PP), da copa (PCOPA), da paleta (PPA), do carré (PC), das costelas (PCOS), da papada (PAPADA) e do filezinho (PF).

Os dados submetidos à análise de correlação canônica foram previamente ajustados para efeitos fixos de sexo, lote e covariáveis. Com base no trabalho de Pires (2003), para ajuste dos dados, foram utilizadas as seguintes covariáveis, para análise das características: tamanho de leitegada à desmama para P77; peso aos 77 dias de idade para CR e CA; peso da carcaça para IDA, PBDIR, CCMB, SH, PROFLOMB, COR, BACO e RIM; e peso da banda carcaça direita resfriada para PP, PCOPA, PPA, PC, PCOS, PAPADA e PF.

Considerando-se o grande número de variáveis medidas em unidades diferentes, foi necessária a padronização dessas variáveis $X_{j}(j=1,2, \ldots . ., p)$. Nesse caso, a estrutura de dependência de $X_{j}$ foi determinada pela matriz de correlação $\mathbf{R}$ (Khattree \& Naik, 2000).

Foram calculadas, inicialmente, duas combinações lineares (variáveis canônicas), uma sobre cada conjunto de variáveis, de forma que os coeficientes de correlação de Pearson entre essas combinações fossem máximos. Em seguida, foram calculadas duas outras variáveis canônicas, uma sobre cada conjunto de variáveis, formando o segundo par canônico, com a condição de que as variáveis canônicas dentro de cada conjunto fossem ortogonais e assim sucessivamente (Johnson \& Wichern, 1998).

Define-se, segundo Regazzi (2002), como a primeira correlação canônica aquela que maximiza a relação entre $U_{1}=a_{1}^{\prime} \underset{\sim}{X}$ e $V_{1}=\underset{\sim}{b_{1}^{\prime}} \underset{\sim}{Y}$. As variáveis canônicas $U_{1} e V_{1}$ constituem o primeiro par canônico associado à primeira correlação canônica, expressa por:

$$
\mathrm{r}_{1}=\frac{\operatorname{Cô}\left(U_{1}, V_{1}\right)}{\sqrt{\hat{V}\left(U_{1}\right) \cdot \hat{V}\left(V_{1}\right)}} .
$$

A porcentagem da variância explicada pela variável canônica $U_{X_{i}}^{2}$ e $V_{Y_{i}}^{2}$ é determinada por:

$$
U_{X_{i}}^{2}=\frac{\sum_{j=1}^{p} a_{i j}^{2}}{p} \text { e } V_{Y_{i}}^{2}=\frac{\sum_{J=1}^{q} b_{i j}^{2}}{q} \text {. }
$$

em que: $p$ e $q$ são os números de variáveis de $X$ e $Y$, respectivamente.

Para verificar a significância da correlação canônica nos pares canônicos, foi utilizado o teste da razão de máxima verossimilhança, cuja estatística é a Lambda $(\Lambda)$ de Wilks, obtido pelo procedimento PROC CANCORR do SAS (1999); cujas expressões são apresentadas por Khattree \& Naik (2000).

\section{Resultados e Discussão}

O número de observações, as médias corrigidas e os desvios-padrão das características de desempenho e de carcaça encontram-se na Tabela 1. 
Os coeficientes padronizados, a correlação canônica, a correlação canônica ao quadrado e o teste estatístico para os pares canônicos entre características de desempenho e de carcaça são apresentados na Tabela 2.

Os grupos características de desempenho e características de carcaça não foram considerados independentes (Tabela 2). Os dois primeiros pares canônicos foram estatisticamente relevantes ( $\mathrm{P}<0,01$ e $\mathrm{P}<0,05$, respectivamente), comprovando que as características de desempenho e de carcaça avaliadas se correlacionam significativamente, pois a correlação do primeiro par canônico (r) foi de 0,7804 $(\mathrm{P}<0,01)$.

Em razão de existir mais de um par canônico estatisticamente relevante, a discussão foi feita para cada par canônico separadamente.
A proporção de variância comum para as duas variáveis canônicas no primeiro par canônico, que é dado pelo coeficiente de correlação canônica ao quadrado $\left(r^{2}\right)$, foi de $60,90 \%$, conforme apresentado na Tabela 2.

O exame das variáveis que compõem o primeiro par canônico apresenta, entre as características de desempenho, predomínio absoluto da variável peso aos 77 dias de idade (P77). Entre as características de carcaça, a variável idade ao abate (IDA) teve predomínio absoluto.

Com o objetivo de auxiliar no procedimento de interpretação, alguns artifícios têm sido desenvolvidos, a fim de ajudar o pesquisador na identificação do tipo de relações representado por um coeficiente de correlação canônica; um desses artifícios é representado pela correlação entre as variáveis originais e as canônicas (Abreu \& Vetter, 1978).

Tabela 1 - Número de observações, médias corrigidas e desvios-padrão das características de desempenho e de carcaça

Table 1 - Number of observations, adjusted means and standard deviation of performance and carcass traits

\begin{tabular}{|c|c|c|c|}
\hline $\begin{array}{l}\text { Característica }^{1} \\
\text { Trait }^{1}\end{array}$ & $\begin{array}{l}\text { Número de observações } \\
\text { Number of observations }\end{array}$ & $\begin{array}{l}\text { Média corrigida } \\
\text { Adjusted mean }\end{array}$ & $\begin{array}{c}\text { Desvio-padrão }^{2} \\
\text { Standard deviation }^{2}\end{array}$ \\
\hline $\operatorname{TLN}(B L)\left(n^{0}\right)$ & 798 & 10,420 & 2,13024 \\
\hline P77 (W77) (kg) & 535 & 21,552 & 3,96670 \\
\hline $\mathrm{CR}(F I)(\mathrm{kg})$ & 595 & 40,299 & 6,52833 \\
\hline $\mathrm{CA}(F G R)(\mathrm{kg} / \mathrm{kg})$ & 580 & 2,802 & 0,62664 \\
\hline CCMB $(B L C)(\mathrm{cm})$ & 527 & 86,128 & 3,30273 \\
\hline $\mathrm{SH}(\mathrm{mm})$ & 526 & 40,346 & 4,78134 \\
\hline PROFLOMB $(L D)(\mathrm{mm})$ & 456 & 44,000 & 4,08628 \\
\hline $\operatorname{COR}(H W)(\mathrm{kg})$ & 526 & 0,236 & 0,02657 \\
\hline $\mathrm{BACO}(S P L W)(\mathrm{kg})$ & 526 & 0,093 & 0,01798 \\
\hline PC $(B L W)(\mathrm{kg})$ & 528 & 3,486 & 0,31579 \\
\hline PCOS $(S P W)(\mathrm{kg})$ & 536 & 1,525 & 0,19546 \\
\hline PAPADA $(J W)(\mathrm{kg})$ & 534 & 0,712 & 0,19139 \\
\hline $\mathrm{PF}(S L W)(\mathrm{kg})$ & 534 & 0,222 & 0,03073 \\
\hline RIM $(K W)(\mathrm{kg})$ & 525 & 0,127 & 0,01646 \\
\hline
\end{tabular}

1 TLN - tamanho da leitegada ao nascimento; NT - número de tetos; P77 - peso aos 77 dias de idade; CR - consumo de ração; CA - conversão alimentar dos 77 aos 105 dias de idade; IDA - idade ao abate; PBDIR - peso da meia-carcaça direita; CCMB - comprimento de carcaça pelo Método Brasileiro de Classificação de Carcaça; SH - maior espessura de toucinho na região da copa, na linha dorso-lombar; PROFLOMB - profundidade de lombo; COR - peso do coração; BACO - peso de baço; PBDIRres - peso da meia-carcaça direita resfriada; PP - peso do pernil; PCOPA - peso da copa; PPA - peso da paleta; PC - peso do carré; PCOS - peso das costelas; PAPADA - peso da papada; PF - peso do filezinho; RIM - peso do rim.

${ }^{1}$ BL - litter size at birth; TN - teat number; W77- weight at 77 days of age; FI - feed intake; FGR - feed/gain rate from 77 to 105 days; SA-slaughter age; $\mathrm{HCW}$ - right half carcass weight; BLC - carcass length by the Brazilian carcass classification method; SH - higher backfat thickness at boston shoulder region, in the midline; LD - loin depth; HW - heart weight; SPLW - spleen weight; CCW - cold right half carcass weight; HAW - ham weight; BSW - boston shoulder weight; PSW - picnic shoulder weight; BLW - loin (bone-in) weight; SPW - spareribs weight; JW - jowl weight; SLW - sirloin weight; and KW - kidney weight.

2 Desvio-padrão dos dados corrigidos para efeitos fixos e covariáveis.

2 Standard deviation of adjusted data to fixed effects and covariate. 
Tabela 2 - Coeficientes padronizados, correlação canônica (r), correlação canônica ao quadrado $\left(r^{2}\right)$ e teste estatístico (F) para os pares canônicos entre características de desempenho e de carcaça

Table 2 - Standardized coefficients, canonical correlation $(r)$, squared canonical correlation $\left(r^{2}\right)$ and statistic test $(F)$ to the canonical variable pairs between performance and carcass traits

\begin{tabular}{|c|c|c|c|c|c|}
\hline \multirow[t]{2}{*}{$\begin{array}{l}\text { Característica }^{1} \\
\text { Trait }^{1}\end{array}$} & \multicolumn{5}{|c|}{$\begin{array}{c}\text { Pares canônicos } \\
\text { Canonical variable pairs }\end{array}$} \\
\hline & $1^{o}$ & $2^{\circ}$ & $3^{\circ}$ & $4^{\circ}$ & $5^{\circ}$ \\
\hline $\operatorname{TLN}(B L)$ & 0,0574 & $-0,0978$ & $-0,1984$ & 0,4378 & 0,8726 \\
\hline NT (TN) & $-0,0222$ & 0,9258 & 0,0417 & 0,3888 & $-0,0793$ \\
\hline P77 (W77) & 0,8058 & $-0,3030$ & $-0,1270$ & 0,4172 & $-0,2907$ \\
\hline $\mathrm{CR}(F I)$ & 0,4321 & 0,1032 & 0,7705 & $-0,3790$ & 0,2789 \\
\hline $\mathrm{CA}(F G R)$ & $-0,3714$ & $-0,2827$ & 0,6670 & 0,5753 & $-0,1152$ \\
\hline IDA (SA) & $-0,8665$ & 0,2012 & 0,0527 & $-0,1387$ & 0,0004 \\
\hline PBDIR (HCW) & $-0,0906$ & $-0,4946$ & 0,0714 & 0,1187 & 0,0135 \\
\hline $\operatorname{CCMB}(B L C)$ & $-0,0489$ & 0,2699 & $-0,4772$ & 0,1523 & $-0,2339$ \\
\hline $\mathrm{SH}$ & 0,0327 & $-0,3301$ & 0,2454 & $-0,1361$ & 0,1821 \\
\hline PROFLOMB $(L D)$ & 0,0078 & 0,2229 & $-0,0231$ & $-0,2363$ & 0,1318 \\
\hline $\mathrm{COR}(H W)$ & $-0,1276$ & $-0,1409$ & 0,1968 & $-0,0897$ & $-0,4514$ \\
\hline $\mathrm{BACO}(S P L W)$ & 0,0306 & $-0,0956$ & $-0,1663$ & 0,0221 & $-0,1309$ \\
\hline PBDIRres (CCW) & 0,4794 & 0,2853 & 0,1828 & $-0,5670$ & $-0,2341$ \\
\hline $\mathrm{PP}(H A W)$ & 0,0605 & $-0,1999$ & $-0,0204$ & 0,1151 & $-0,2085$ \\
\hline PCOPA (BSW) & 0,0944 & 0,0624 & $-0,2023$ & 0,0600 & 0,4894 \\
\hline PPA (PSW) & 0,0167 & $-0,3201$ & $-0,2455$ & 0,2547 & $-0,1842$ \\
\hline $\mathrm{PC}(B L W)$ & 0,0540 & 0,3778 & 0,2739 & 0,7713 & $-0,1946$ \\
\hline PCOS (SPW) & 0,0608 & 0,5194 & $-0,0917$ & $-0,0863$ & 0,2531 \\
\hline PAPADA $(J W)$ & $-0,0249$ & $-0,1178$ & 0,0193 & 0,2908 & 0,2735 \\
\hline $\mathrm{PF}(S L W)$ & 0,0267 & $-0,2605$ & $-0,2691$ & $-0,0691$ & 0,2978 \\
\hline $\operatorname{RIM}(K W)$ & 0,0585 & 0,1802 & 0,2044 & 0,1900 & 0,1262 \\
\hline $\mathrm{r}$ & 0,7804 & 0,3370 & 0,2607 & 0,1930 & 0,1249 \\
\hline $\mathrm{F}$ & $5,45 * *$ & $1,37 *$ & $0,98 \mathrm{~ns}$ & $0,68 \mathrm{~ns}$ & $0,43 \mathrm{~ns}$ \\
\hline $\mathrm{r}^{2}$ & 0,6090 & 0,1136 & 0,0680 & 0,0372 & 0,0156 \\
\hline
\end{tabular}

1 Conforme descrito na Tabela 1.

* Significativo a $5 \%(P<0,05)$.

** Significativo a $1 \%(P<0,01)$.

ns Não-significativo a $5 \%(P>0,05)$.

1 According to Table 1.

* Significant at $5 \%(P<0.05)$.

** Significant at $1 \%(P<0.01)$.

ns Not significant at $5 \%(P>0.05)$.

No exame de correlação entre a variável original e sua correlação canônica, confirma-se que P77 é a mais importante na variável canônica $U_{1}$ e que a variável IDA é a mais importante na variável canônica $\mathrm{V}_{1}$ (Tabela 3). Portanto, maior peso aos 77 dias de idade (característica de desempenho) proporciona menor idade ao abate (característica de carcaça), ressaltando-se que, em muitos casos, interpretações com base nos coeficientes padronizados e nas correlações entre as variáveis originais e as canônicas podem ser muito diferentes.

Com base na Tabela 3, foram obtidos os valores de: $U_{X_{1}}^{2}=0,2079$ e $V_{Y_{1}}^{2}=0,0643$

Assim, 20,79\% da variância das características de desempenho é explicada pela variável canônica $U_{1}$ e 6,43\% da variância das características de carcaça, pela variável canônica $\mathrm{V}_{1}$.
A proporção de variância comum às duas variáveis canônicas no segundo par canônico, determinada pelo coeficiente de correlação canônica ao quadrado $\left(r^{2}\right)$, foi de $11,36 \%$ (Tabela 2).

O exame das variáveis que compõem o segundo par canônico apresenta, entre as características de desempenho, predomínio absoluto da variável número de tetos (NT). Entre as características de carcaça, a variável peso das costelas (PCOS) teve a maior contribuição, seguida pelo peso da meia-carcaça direita (PBDIR) (Tabela 2).

No exame de correlação entre a variável original e sua correlação canônica, confirmou-se que a variável NT é a mais importante na variável canônica $\mathrm{U}_{2}$ e que a variável PCOS é a mais importante na variável canônica $\mathrm{V}_{2}$ (Tabela 3). Portanto, quanto maior o número de tetos (características de desempenho), maior o peso das costelas (características de carcaça). 
Com base na Tabela 3, foram também obtidos os valores de: $U_{X_{2}}^{2}=0,1935$ e $V_{Y_{2}}^{2}=0,05196$.

Desse modo, 19,35\% da variância das características de desempenho é explicada pela variável canônica $U_{2}$ e 5,20\% da variância das características de carcaça, pela variável canônica $\mathrm{V}_{2}$.

\section{Conclusões}

Os conjuntos de características de desempenho e de carcaça são considerados dependentes.

Entre as variáveis avaliadas, P77, NT, IDA, PCOS e PBDIR foram consideradas as mais importantes para explicar a associação entre as características de desempenho e de carcaça.

Tabela 3 - Correlação entre as características de desempenho e suas variáveis canônicas (U) e entre as características de carcaça e suas variáveis canônicas (V)

Table 3 - Correlation between the performance traits and their canonical variables (U) and among the carcass traits and their canonical variables $(V)$

\begin{tabular}{|c|c|c|c|c|c|}
\hline $\begin{array}{l}\text { Característica } \\
\text { Trait }^{1}\end{array}$ & $\mathrm{U}_{1}$ & $\mathrm{U}_{2}$ & $\mathrm{U}_{3}$ & $\mathrm{U}_{4}$ & $\mathrm{U}_{5}$ \\
\hline $\mathrm{TLN}(B L)$ & 0,0717 & $-0,0749$ & $-0,1676$ & 0,3844 & 0,9019 \\
\hline $\mathrm{NT}(\mathrm{TN})$ & 0,0986 & 0,8981 & 0,0629 & 0,4124 & $-0,0984$ \\
\hline P77 (W77) & 0,8027 & $-0,1955$ & $-0,0838$ & 0,4548 & $-0,3219$ \\
\hline $\mathrm{CR}(F I)$ & 0,4804 & 0,1613 & 0,7076 & $-0,3650$ & 0,3305 \\
\hline CA (FGR) & $-0,3870$ & $-0,3019$ & 0,6120 & 0,5968 & $-0,1686$ \\
\hline IDA $(S A)$ & $\begin{array}{c}V_{1} \\
-0,8614\end{array}$ & $\begin{array}{c}\mathrm{V}_{2} \\
0,1591\end{array}$ & $\begin{array}{c}V_{3} \\
0,1260\end{array}$ & $\begin{array}{c}V_{4} \\
-0,2493\end{array}$ & $\begin{array}{c}V_{5} \\
-0,0370\end{array}$ \\
\hline PBDIR (HCW) & 0,0674 & $-0,3174$ & 0,0191 & 0,1371 & 0,1922 \\
\hline CCMB (BLC) & $-0,0072$ & 0,2852 & $-0,6674$ & 0,2210 & $-0,2952$ \\
\hline SH & $-0,0740$ & $-0,2383$ & 0,5781 & 0,0054 & 0,1571 \\
\hline PROFLOMB (LD) & $-0,0306$ & 0,2243 & $-0,0822$ & $-0,1786$ & 0,0671 \\
\hline $\mathrm{COR}(H W)$ & $-0,2023$ & $-0,0995$ & $-0,0577$ & $-0,0794$ & $-0,4714$ \\
\hline $\mathrm{BACO}(S P L W)$ & $-0,0037$ & $-0,0602$ & $-0,1782$ & 0,0013 & $-0,2480$ \\
\hline PBDIRres (CCW) & 0,4621 & 0,1996 & 0,2002 & $-0,5298$ & $-0,2298$ \\
\hline $\mathrm{PP}(H A W)$ & 0,0512 & $-0,2616$ & 0,0258 & 0,1401 & $-0,3652$ \\
\hline PCOPA (BSW) & 0,0589 & $-0,0666$ & $-0,3380$ & 0,1476 & 0,3547 \\
\hline $\operatorname{PPA}(P S W)$ & 0,0281 & $-0,1642$ & $-0,5794$ & $-0,0346$ & $-0,2788$ \\
\hline PC $(B L W)$ & 0,0704 & 0,2649 & 0,4345 & 0,6268 & $-0,1378$ \\
\hline $\operatorname{PCOS}(S P W)$ & 0,0357 & 0,4583 & $-0,2434$ & $-0,0648$ & 0,2849 \\
\hline PAPADA (JW) & 0,0838 & 0,0079 & 0,1698 & 0,1029 & 0,3518 \\
\hline $\mathrm{PF}(S L W)$ & $-0,0146$ & $-0,2158$ & $-0,3530$ & $-0,1376$ & 0,2638 \\
\hline $\operatorname{RIM}(K W)$ & 0,0375 & 0,1919 & 0,1232 & 0,1156 & 0,1357 \\
\hline
\end{tabular}

${ }^{1}$ Conforme descrito na Tabela 1.

${ }^{1}$ According to Table 1.

\section{Literatura Citada}

ABREU, M.A.; VETTER, D.A. Análise de relação entre conjuntos de variáveis na matriz geográfica: correlação Canônica. In: FAISSOL, S. (Ed.) Tendências atuais na geografia urbano/regional: teorização e quantificação. Rio de Janeiro: IBGE, 1978. p.133-144.

ALMEIDA NETO, P.P.; OLIVEIRA, A.I.G.; ALMEIDA, A.J.L. et al. Parâmetros genéticos e fenotípicos de características de carcaça de suínos. Revista Brasileira de Zootecnia, v.22, p.624-633, 1993.

BARBOSA, L. Utilização de técnicas de análise multivariada na avaliação de características quantitativas de uma população F2 de suínos. Viçosa, MG: Universidade Federal de Viçosa, 2003. 90p. Dissertação (Mestrado em Zootecnia) - Universidade Federal de Viçosa, 2003.

COSTA, A.R.C.; LOPES, P.S.; TORRES, R.A. et al. Estimação de parâmetros genéticos em características de desempenho de suínos das raças Large White, Landrace e Duroc. Revista Brasileira de Zootecnia, v.30, p.49-55, 2001.

DUFOR, E.; DEVAUX, M.F.; FORTIER, R. et al. Delineation of the structure of soft chesses at the molecular level by fluorescence spectroscopy - relationship with texture. International Dairy Journal, v.11, p.465-473, 2001.

FRANCI, O.; PULGLIESE, C.; BOZZI, R. et al. The use of multivariate analysis for evaluanting relationships among fat depots in heavy pigs of different genotypes. Meat Science, v.58, p.259-266, 2001.

JOHNSON, R.A.; WICHERN, D.W. Applied multivariate statistical analysis. 4. ed. Englewood Cliffs: Prentice Hall, 1998. 816p.

KHATTREE, R.; NAIK, D.N. Multivariate data reduction and discrimination with SAS software. New York: BBU Press and John Wiley Sons Inc., 2000. 574p.

LIBERATO, J.R. Aplicação de técnica de análise multivariada em fitopatologia. Viçosa, MG: Universidade Federal de Viçosa, 1995, 144p. Dissertação (Mestrado em Fitopatologia) Universidade Federal de Viçosa, 1995. 
LIBERATO, J.R.; VALE, F.X.R.; CRUZ, C.D. Técnicas estatísticas de análise multivariada e a necessidade de o fitopatologista conhecê-las. Fitopatologia Brasileira, v.24, p.5-8, 1999.

MONTGOMERY, D.C.; PECK, E.A. Introduction to linear regression analysis. 2.ed. New York: John Wiley Sons, 1992. 527p.

NATHIER-DUFOUR, N.; ANGUE, Y.; DEVAUX, D.B. et al. Influence of wheat meal variability upon compacting behavior during pelleting. Animal Feed Science and Technology, v.51, p.255-268, 1995 .

PIRES, A.V. Mapeamento de locos de características quantitativas, no cromossomo seis suíno. Viçosa, MG: Universidade Federal de Viçosa, 2003, 86p. Tese (Doutorado em Genética e Melhoramento) - Universidade Federal de Viçosa, 2003.

REGAZZI, A.J. Análise multivariada. Viçosa, MG: Universidade Federal de Viçosa, 2002. (INF-766) (notas de aula).

ROSO, V.M.; FIRES, L.A.; MARTINS, E.S. Parâmetros genéticos em características de desempenho e qualidade de carcaça em suínos da raça duroc. Revista Brasileira de Zootecnia, v.24, p.310-316,1995.

SAKAGUTI, E.S. Utilização de técnicas de análise multivariada na avaliação de cruzamento dialélicos em coelhos. Viçosa, MG: Universidade Federal de Viçosa, 1994, 170p. Dissertação (Mestrado em Genética e Melhoramento) - Universidade Federal de Viçosa, 1994.

SAKAGUTI, E.S.; SILVA, M.A.; REGAZZI, A.J. et al. Avaliação dos testes da análise de variância multivariada em experimentos com dados desbalanceados. Revista Brasileira de Zootecnia, v.25, p.437-448, 1996.
STATISTICAL ANALYSES SYSTEM - SAS. Software: versão 8.0. Cary: 1999.

SILVA, M.A.; CATALAN, G.; TORRES, R.A. et al. Estimativas de componentes genéticos de características de importância econômica, em três diferentes raças de suínos. Revista Brasileira de Zootecnia, v.25, p.923-32, 1992.

TORRES FILHO, R.A. Avaliação genética de características de desempenho e reprodutivas em suínos. Viçosa, MG: Universidade Federal de Viçosa, 2001. 81p. Dissertação (Mestrado em Genética e Melhoramento) - Universidade Federal de Viçosa, 2001.

TORRES JR., R.A.A.; SILVA, M.A.; LOPES, P.S. et al. Estimativas de componentes de (co)variância para características produtivas de suínos Landrace e Large White pelo método da máxima verossimilhança restrita. Revista Brasileira de Zootecnia, v.27, p.283-91, 1998.

VAINIONPÃÃ, J.; KERVINEN, R.; De PRADO, M. et al. Exploration of storage and process tolerance of different potato cultivars using principal component and canonical correlation analyses. Journal of Food Engineering, v.44, p.47-61, 2000.

Recebido em: 24/09/05

Aceito em: 29/06/05 